\title{
Layer-number-dependent Optical and Electrical Properties of Graphene on $\mathrm{ZnO}$
}

\author{
Yi-Hang $\mathrm{Ku}^{1,2}$, Lo-Yueh Chang ${ }^{1}$, Hung-Wei Shiu ${ }^{1}$, Yen-Chien Kuo ${ }^{1}$, Shangjr Gwo ${ }^{1,2}$, Chi-Liang \\ Chen $^{1 *}$, and Chia-Hao Chen ${ }^{1 *}$ \\ 1. National Synchrotron Radiation Research Center, Hsinchu, 30076, Taiwan \\ 2. Department of Physics, National Tsing-Hua University, Hsinchu, 30013, Taiwan \\ * Corresponding authors, chen.cl@nsrrc.org.tw (CLC) and chchen@nsrrc.org.tw (CHC)
}

Metal/semiconductor (MS) junction is an essential element for the electronics applications [1-4]. The junctions can either show Ohmic behavior or form Schottky diode contact; both are parts of the basic building blocks for the semiconductor devices. As is well known, device performance largely depends on the quality of the MS contacts. It is therefore important to study the electronic structure of various MS hybrid systems. In the past decades, the wide band gap semiconductors have attracted considerable amount of interest, because of their superior optoelectronic properties and partially due to problem to find Ohmic contact materials matching their wide energy gap. Among these wide band gap semiconductors, because of its unique excitonic, photonic, and electronic properties, zinc oxide ( $\mathrm{ZnO}$, band gap $\sim 3.3 \mathrm{eV}$ ) has been intensively studied over the past few years [5]. On the other hand, graphene is prospectively a favorable material for future electronic applications because of its surprising physical and chemical properties; the high electron mobility and heat conductance, and its optically transparency [6]. Combining these attractive properties of both materials is expected for the next generation optoelectronic devices, because they are easy to fabricate, low-cost, non-toxic and high electric efficiency [5]. Therefore, the fundamental knowledge of graphene/ $\mathrm{ZnO}$ interface is important and will lead to improved understanding of the intrinsic electronic properties of the graphene/semiconductor hybrid systems.

In this regard, we have carried out the study of optical and interfacial electronic properties between graphene layers and $n$-type $\mathrm{ZnO}$. The $\mathrm{ZnO}$ films $(\sim 12 \mathrm{~nm})$ were synthesized on $\mathrm{Si}$ substrates with $\mathrm{SiO}_{2}$ $(\sim 66 \mathrm{~nm})$ as a buffer layer by atomic layer deposition. Single-layer graphene (SLG), bi-layer graphene (BLG), and multi-layer graphene (MLG) samples were prepared by mechanical exfoliation from highly ordered pyrolytic graphite on $n$-type $\mathrm{ZnO}$ film. The optimal optical visibility of graphene layers was simulated based on Fresnel's law [7] and confirmed with an optical microscope (OM) and the microRaman spectra, as shown in Fig. 1. The interfacial electronic property was studied by scanning photoelectron microscope (SPEM) locates at beamline 09A1 in National synchrotron radiation Research Center (NSRRC), Taiwan. The SPEM measurements were conducted at room temperature with $380 \mathrm{eV}$ $\mathrm{X}$-ray photon energy. The photon energy was calibrated by measuring the $\mathrm{Au} 4 f$ core level line emitted from a clean gold foil electrically contacted with the samples [8].

Figure 2 shows the C $1 s$ SPEM image taken from the same location as the OM image showed in Fig. 1 (a). Both images are almost identical. By carefully analyzing the positions of the $\mathrm{C} 1 s$ and $\mathrm{Zn} 3 d$ photoelectron peaks and the valence band maximum (VBM) locations from graphene with various thicknesses, we found that the VBM of SLG on ZnO surface is located $0.7 \mathrm{eV}$ below the Dirac point, which makes it a $p$-type semiconductor for graphene on $\mathrm{ZnO}$ substrate. Since the $\mathrm{ZnO}$ is an $n$-type semiconductor, our results show that the $\mathrm{SLG} / \mathrm{ZnO}$ heterostructure will naturally form a $p$ - $n$ 
heterojunction, which is difficult to achieve for $\mathrm{ZnO}$ based heterostructures.

Furthermore, the binding energy of the $\mathrm{Zn} 3 d$ remains constant while the graphene thickness varied from bare $\mathrm{ZnO}$ surface to MLG. This phenomenon implies a flat band condition for the graphene layer contacts with $\mathrm{ZnO}$, which may lead to a superior transparent electrical contact for $\mathrm{ZnO}$.

In summary, we have demonstrated the visibility and band alignment of graphene on an $n$-type $\mathrm{ZnO}$ substrate. Applying Fresnel theory, a specific substrate layer structure and thickness were found to achieve a maximum optical contrast. Deduced from the C $1 s$, Zn $3 d$ and VBM positions, we can conclude that the $\mathrm{ZnO}$ substrate induced a $p$-type doping on the single-layer graphene overlayer, and the graphene/ $n$-type $\mathrm{ZnO}$ formed a flat band interface, which is independent of the graphene thickness. The combined result indicated that the graphene will form a $p-n$ heterojunction, and will lead to be an ideal transparent electrode while contact with $n$-type $\mathrm{ZnO}$ [9].

\section{References:}

[1] R. T. Tung, Phys. Rev. B 45, 13509 (1992).

[2] L. J. Brillson, and Y. Lu, J. App. Phys., 109, 121301 (2011).

[3] B. L. Sharma, Metal-Semiconductor Schottky Barrier Junctions and Their Applications, SpringerVerlag New York Inc. (2012).

[4] T. Mueller, F. Xia, and P. Avouris, Nature Photonics 4, 297 (2010).

[5] S. W. Hwang et al., Phys. Rev. Lett. 105, 127403 (2010).

[6] E. H. Hwang et al., Phys. Rev. Lett. 98, 186806 (2007).

[7] H. W. Shiu et al., App. Phys. Lett. 103, 081604 (2013).

[8] H. W. Shiu et al., J. App. Phys. 113, 043701 (2013).

[9] The authors thank the NSRRC beamline staff for their skillful assistance during the experiments. This work was partially financial supported by the Ministry of Science and Technology of Taiwan.
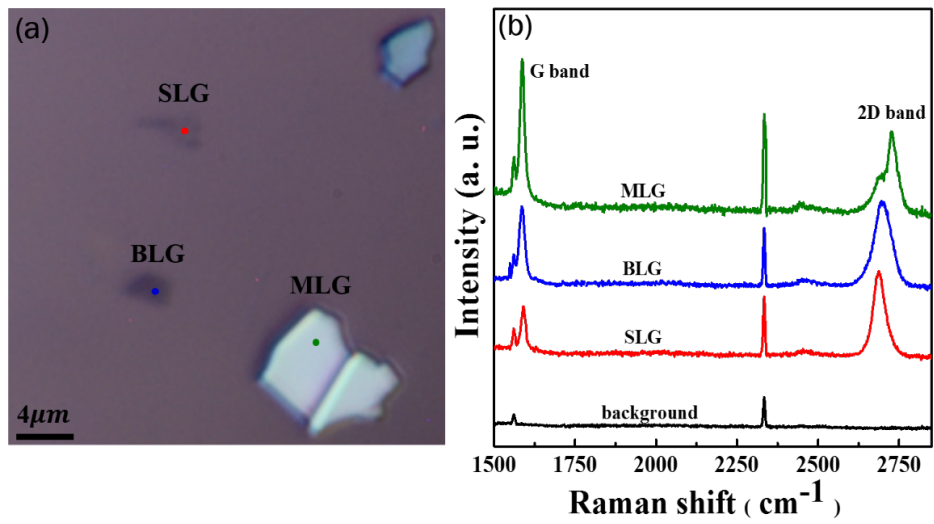

Fig. 1. (a) OM images of graphene with varied number of layers on $\mathrm{ZnO}$ surface. (b) Raman spectra recorded in regions of bare $\mathrm{ZnO}$ (background), SLG (red), BLG (blue) and MLG (green). The wavelength of the incident laser beam is $532 \mathrm{~nm}$.

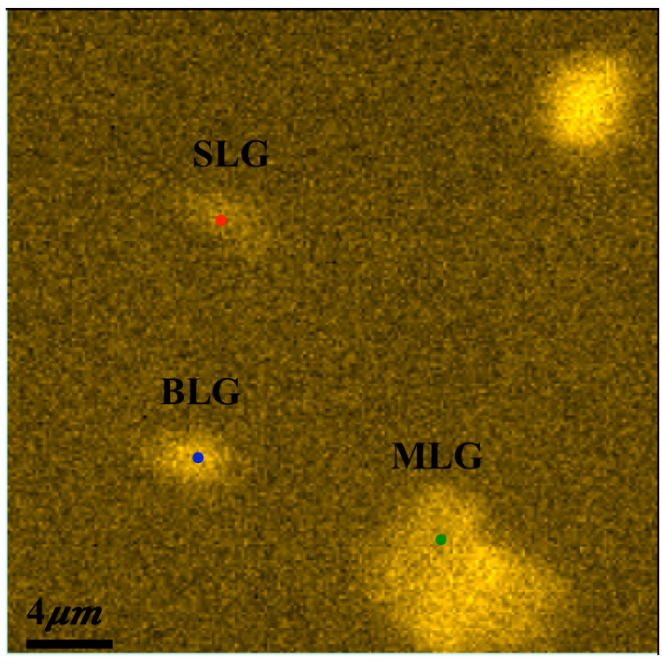

Fig. 2. The SPEM C 1s image taken from the same location as the $\mathrm{OM}$ image showed in Fig. 1 (a). 